\title{
UCRL-TR-209699
}

LAWRENCE LIVERMORE N A TION A L LABORATORY

Edge cladding gain media according to IL-11317

Thomas Soules

February 14, 2005 
This document was prepared as an account of work sponsored by an agency of the United States Government. Neither the United States Government nor the University of California nor any of their employees, makes any warranty, express or implied, or assumes any legal liability or responsibility for the accuracy, completeness, or usefulness of any information, apparatus, product, or process disclosed, or represents that its use would not infringe privately owned rights. Reference herein to any specific commercial product, process, or service by trade name, trademark, manufacturer, or otherwise, does not necessarily constitute or imply its endorsement, recommendation, or favoring by the United States Government or the University of California. The views and opinions of authors expressed herein do not necessarily state or reflect those of the United States Government or the University of California, and shall not be used for advertising or product endorsement purposes.

This work was performed under the auspices of the U.S. Department of Energy by University of California, Lawrence Livermore National Laboratory under Contract W-7405-Eng-48. 
September 17, 2004

\section{Edge cladding gain media according to IL-11317}

\section{Elements claimed}

In this patent application we wish to claim the following approach to ameliorating spontaneous amplified emission (ASE) that occurs in a laser amplifier slab. There are two important elements of our approach. We wish to claim the application of both together but not either one alone.

1. The first element of the invention is to roughen the edge surfaces of the amplifier slab. A rough surface with random planar features larger than the wavelength of light will reflect and refract incident light rays at angles different than the median plane of the surface. The rough surface can then be characterized by two parameters. First there is a distribution of heights about the zero mean plane of the surface. If normal this distribution is characterized by a standard deviation. The second parameter is the correlation distance that describes how close together on average are the peaks and valleys. The ratio of these two numbers determines the spread of light reflected off the surface of the edge of the slab.

The purpose of roughening the edge is to prevent ASE from being internally reflected at a prescribed angle so that it can establish an infinite path inside the crystal. Such a ray would be amplified in preference to the desired beam and would become parasitic and would clamp the gain. This is illustrated below.

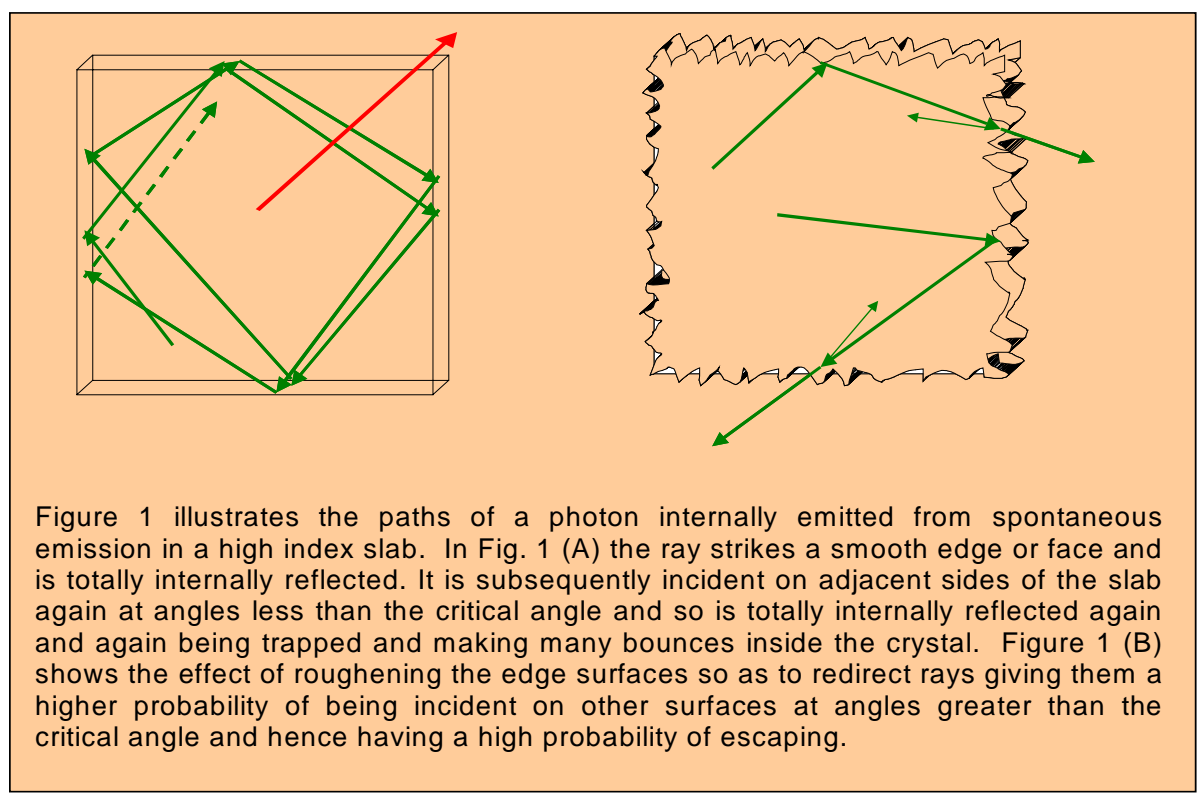


The edge surface can be roughened, such that a beam of light striking the edge surface will be spread out from the specular angle over a Gaussian cone. Preferably the half intensity of the beam spread would be between 5 and 55 degrees, more preferably between 5 and 30 degrees, most preferably between 5 and 15 degrees.

There are many ways to achieve the rough edges described above and we have performed experiments using several of these. We have used standard polishing techniques on our GGG:Nd amplifier slabs on the edges with 30 and 45 micrometer sized alumina grinding media was to achieve a rough surface and 5 and 10 micrometer grinding media for a smoother finish. We also prepared edges using a rough blanchard wheel, a smooth blanchard wheel, and a medium grinding wheel. There are many other techniques commonly used in industry to achieve surfaces of different degrees of roughness. These techniques would be covered if they achieved the above mentioned optical reflection characteristics. We have profilometer trace curves for those techniques that we have tried. Several of these methods of roughening the surface appear to work equally well when used in laser gain experiments.

2. The second element in our invention is to bond the roughened edges of the gain medium to an ASE absorbing media using a suitable bonding agent. In order for the ASE to leave the gain medium crystal there must be minimal reflection and maximum transmission at the interface. This requires having a near match of the index of refraction of the bonding medium and the amplifier slab material at the wavelength of the ASE. Further if the index of refraction of the bonding agent is less than that of the amplifer there will be some total internal reflection even if the surface is roughened. The index of refraction of GGG is $\sim 1.92$ and that of YAG is $\sim 1.84$. There are no suitable bonding agents with indices of refraction this high.

However, we have carried out Monte-Carlo calculations that show that using our roughened edges allows us to consider lower indices of refraction and still not get parasitic lasing at the power extractions we desire. This is illustrated in the figure below.

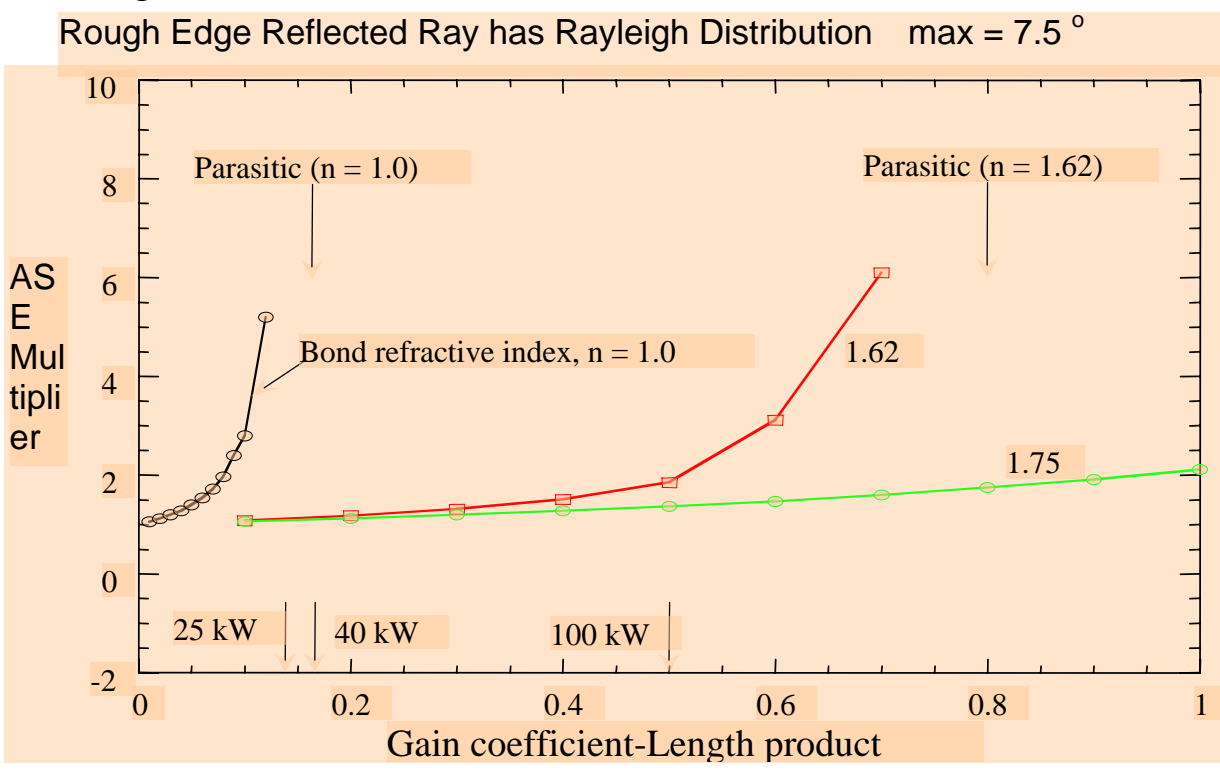


We claim a bonding agent with an index of refraction prefereably greater than 1.5, more preferably greater than 1.6 and most preferably greater than 1.7 .

In an effort to achieve a bonding agent with an index approximately equal to or greater than 1.7 we carried out experiments with the following:

\begin{tabular}{|c|c|c|}
\hline Bonding agent & Index & Issues \\
\hline Titania sol & $>1.65$ & Did not bond \\
\hline $\begin{array}{l}\text { Adding titanium } \\
\text { isopropoxide and/or titania } \\
\text { sol to a polyurethane or } \\
\text { silicone }\end{array}$ & $>1.65$ & $\begin{array}{l}\text { Unable to add sufficient titania to move } \\
\text { the index from } \sim 1.4 \text { to }>1.6 \text { to achieve } \\
\text { high index }\end{array}$ \\
\hline $\begin{array}{l}\text { Pentabromophenyl } \\
\text { methacrylate and similar } \\
\text { compounds }\end{array}$ & $\sim 1.7$ & $\begin{array}{l}\text { Unstable at high temperatures - } \\
\text { bromine gas evolution - poor bonding }\end{array}$ \\
\hline $\begin{array}{l}\text { Added titania nano- } \\
\text { particles to urethane and } \\
\text { silicone }\end{array}$ & & $\begin{array}{l}\text { Too much light scattering due to } \\
\text { agglomeration of nanoparticles }\end{array}$ \\
\hline $\begin{array}{ll}\text { Polyimides - } & \text { Kapton } \\
\text { double sided tape } & \\
\end{array}$ & 1.7 & $\begin{array}{l}\text { Optical contact not established on both } \\
\text { sides - poor bonding }\end{array}$ \\
\hline $\begin{array}{l}\text { Ultem } 1000 \text { sheet - heated } \\
\text { and pressed }\end{array}$ & 1.68 & $\begin{array}{l}\text { Contraction during cooling often } \\
\text { delaminates bond in some areas }\end{array}$ \\
\hline $\begin{array}{l}\text { Ultem sheet dissolved in } \\
\text { dichloromethane and } \\
\text { applied and dried }\end{array}$ & 1.68 & $\begin{array}{l}\text { Bubbles form when solvent is removed. } \\
\text { This was a universal issue when high } \\
\text { index materials were used with solvent }\end{array}$ \\
\hline $\begin{array}{l}\text { Nitto Denko highest } \\
\text { refractive index polyimide }\end{array}$ & 1.76 & $\begin{array}{l}\text { Same issues as with Ultem - poor } \\
\text { bonding when used as thermal plastic }\end{array}$ \\
\hline $\begin{array}{l}9 \text { different organo-metallic } \\
\text { titania containing coupling } \\
\text { agents with/without Ultem }\end{array}$ & ? & Poor bonding \\
\hline
\end{tabular}

Two part epoxies have the advantages of providing very good bonding and have no solvents. For this reason we focused on finding a high temperature, high refractive index epoxy. Two part epoxies consist of a linear polymer usually containing bis-biphenol groups and terminated on either end with epoxide groups. Four epoxide groups react with a diamine curing agent to create a tough in two dimensional polymer molecule whose polar groups bond strongly to the surfaces. Because there is no solvent evolution, no bubbles are created during curing. A typical molecular epoxide structure and the epoxy curing is illustrated below.<smiles>CCc1ccc(C(C)(C)c2ccc(OCC(O)COc3ccc(C(C)(C)c4ccc(OCC5CO5)cc4)cc3)cc2)cc1</smiles> 


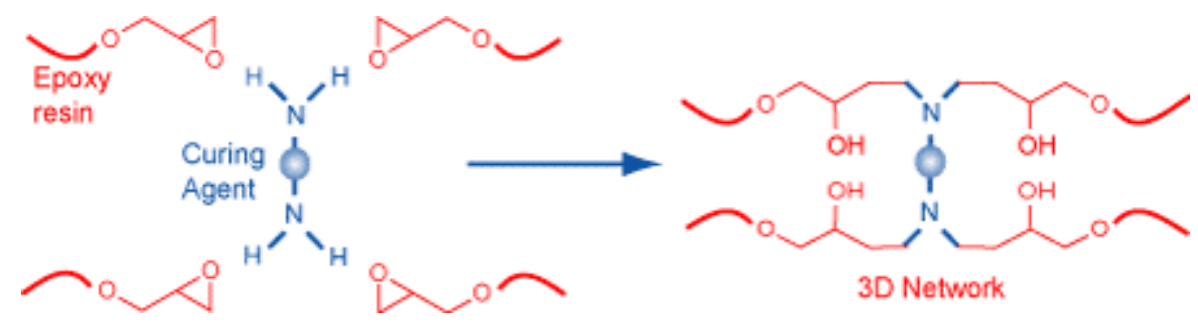

By modifying the structure of the epoxide and the amine curing agent including more conjugated phenol rings one can increase the index of refraction from nominally 1.5 to $>1.6$.

The highest index of refraction commercial epoxies that we found were made by Master Bond Inc. These are also very stable at high temperatures no doubt a consequence of the stability of the conjugated ring structure. Three high index two part epoxies were recommended by Dr. Walter Brenner of Master Bond (1-201-343-8983) Hackensack, NJ. Dr. Brenner had a wealth of information on epoxies including indices of refraction not included in the usual spec sheets. The epoxies he recommended are EP42HT $(n=1.608)$, EP42HT-2 $(\mathrm{n}=1.614)$ and EP45HT $(\mathrm{n}=1.63)$. All are two part epoxies. EP45HT requires heating to $150 \mathrm{C}$ to cure while the others cure at room temperature over approximately 24 hours. The compositions are proprietary but EP42HT is stable to $435 \mathrm{~F}$, EP42HT-2 to $450 \mathrm{~F}$ and EP45HT to 500F. Because it cures at room temperature and because no difference was observed in the laser clamping behavior when EP45HT was used we focused primarily on EP42HT-2 which proved satisfactory -- tough and resistant to not only thermal cycling but also showed no effect from direct bombardment from our diode arrays at $880 \mathrm{~nm}$. Details of the latter experiments are available from Kurt Cutter (2-0190).

\section{Recipe's used in two preferred embodiments}

A. In the first embodiment the roughened edge surfaces of the slab are bonded to the ASE cladding material which may or may not be roughened but which should have an index of refraction greater than that of the epoxy so as to avoid total internal reflection at the epoxy ASE boundary. Preferably the index of refraction of the ASE absorber would be the same as the epoxy. In fact, we have used Co:GGG $(n=1.94)$ and KG3 $(n=1.54)$. KG3 is an infrared absorbing glass made by Schott Inc. but since its index of refraction is less than that of the epoxy it resulted in clamping earlier than with the GGG. The Co:GGG has the advantage of being relatively transparent to the diode radiation at $880 \mathrm{~nm}$. Hence the Co:GGG is heated only by the ASE. The Co concentration is chosen so that this heating is relatively uniform throughout and the cladding maintains a temperature close to that of the lasing amplifier so as not to induce excessive thermal gradients near the edge of the laser amplifier which will lead to wavefront distortion. 
The set up is illustrated below:

1. The edge of the crystal slab is roughened using one of the methods described above. The adjacent face of the edge cladding may be either roughened or polished smooth.

2. Both faces of the amplifier slab are masked.

3. The edge surfaces on both the amplifier slab and adjacent edge cladding pieces are cleaned with acetone following by isopropyl alcohol.

4. The slab edge surfaces and adjacent edge cladding surfaces are then coated with a coupling agent as follows.

i. Carefully wipe the surfaces with a solution of $0.2 \mathrm{~N}$ acetic acid.

ii. Rinse surfaces by wiping with distilled water.

iii. Carefully wipe the surface with a solution of acetic acid, methanol and a silane Z2060. Details of these solutions are available from NIF (L. Auyang 2-9177)..

iv. Rinse surface by wiping with distilled water.

5. Make up mixture of epoxide EP42HT-2 Part A (100 parts by weight) and amine curing agent Part B (40 parts). Thoroughly mix in test tube with clean stirring rod. Centrifuge for 3 minutes to remove bubbles.

6. A small bead of the epoxy mixture is applied to the lower part of the edge cladding.

7. The edge cladding piece is brought up to the edge of the slab so as force the bead to slowly spread out and thouroughly wet both surfaces without creating any bubbles.

8. A bead of epoxy is then similarly applied to the surface of the second edge cladding piece and it is attached and so forth.

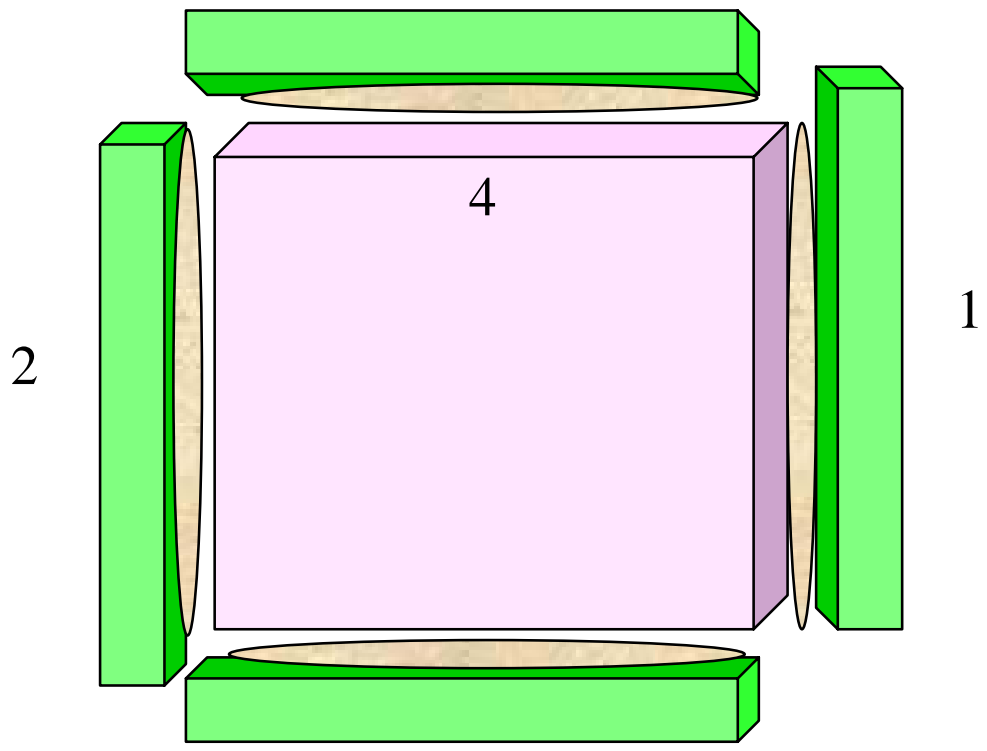


9. The assembly of bonded pieces is placed in a suitable fixture and the sides are clamped lightly in place allowing for expansion joints at each corner. The fixture design and drawings are available from Charles Parks (4-5619). The assembly is set aside for $\sim 24$ hours to cure. The final assembly is illustrated below.

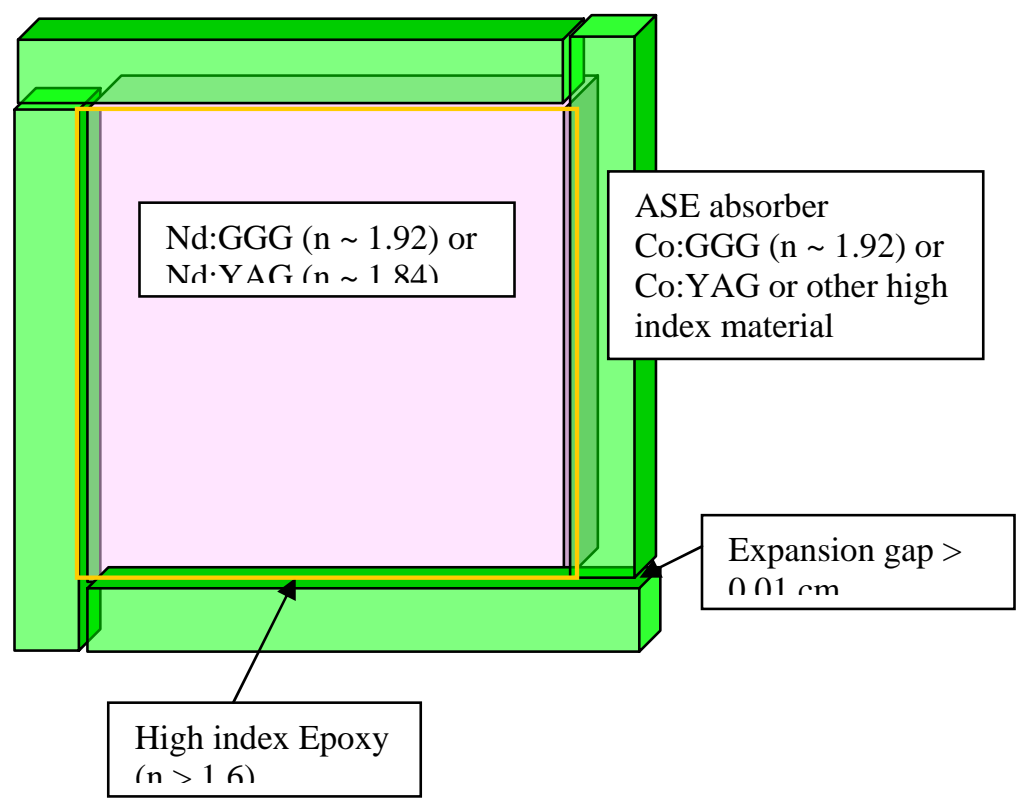

B. In the second preferred embodiment a "moat" of epoxy is poured around the laser amplifier slab between the slab and a frame. The frame can be metal is designed to absorb the ASE. The thick "moat" of epoxy is designed to thermally and mechanically isolate the slab from the ASE absorbing cladding. This allows the cladding to be made of a material which will strongly absorb the ASE on its surface. By making the ASE out of a metal heat can be rapidly removed from the surface. The metal must be "blackened" to absorb and not reflect ASE back into the epoxy and slab. Blackening should involve either a metallic or metal oxide blackening not painting so that the index of refraction change at the surface is from low to high and one avoids ASE reflection back into the epoxy and amplifier slab. A metallic or metal oxide coted metallic ASE absorber clearly has many other advantages, such as, availability, ease of machining and flexibility of design. The design can even include optional cooling channels or channels designed to heat and cool during operation so as to maintain minimal thermal gradients in the amplifier slab. A picture illustrating this second preferred embodiment is shown below. 


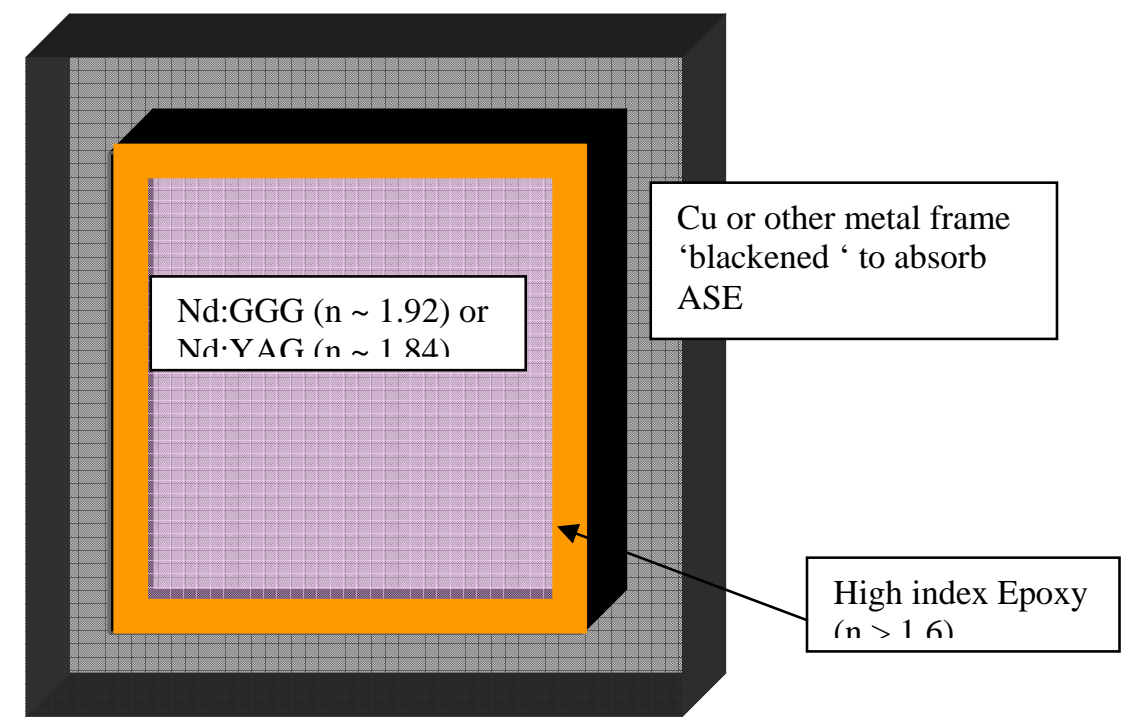

The procedure is outlined as follows:

1. The edge of the crystal slab is roughened using one of the methods described above. The adjacent face of the edge cladding may be either roughened or polished smooth.

2. Both faces of the amplifier slab are masked.

3. A frame opening allowing for a $2 \mathrm{~mm}$ "moat" or gap around the slab is cut from a piece of copper machined to the same thickness as the slab. The copper forms a frame around the slab and may have expansion slots cut at the corners or elsewhere to further alleviate any stress on the slab from differential thermal expansion of the metal relative to the slab.

4. The copper, which may be annealed or hardened, is then treated as follows to create a black ASE absorbing oxide surface.

i. Carefully clean the copper frame with an alkaline cleaning solution..

ii. Rinse in a citric acid solution to further remove any oxide

iii. Dip into a hot, 200F, blackening solution made with Ebonal C. Ebanol $\mathrm{C}$ is a proprietary mixture of sodium hydroxide and sodium chlorite from Enthone Corporation. It produces a black tenacious cupric oxide on the surface of the copper

iv. Wipe excess black fuzz off the surface.

v. Wash in a hot DI water ultrasonic bath and dry.

5. The slab is then positioned to be in the middle of the metal frame and clamped down against a soft neoprene rubber to seal off the bottom. The fisture for doing this was designed and built by C. Parks (4-5619).

6. The EP42HT-2 epoxy is then prepared as above and centrifudged to remove any bubbles. 
7. The epoxy is introduced into the $2 \mathrm{~mm}$ wide moat between the slab and the metal frame with a hypodermic syringe taking care not to introduce any bubbles. The moat around the slab is filled and an excess convex bead is added to the top to account for contraction during curing. A second bead may have to be added after or during curing to account for shrinkage.

8. The epoxy is then left at room temperature to cure for 24 hours.

It is clear that there are many variations of the above embodiments that would be considered to be obvious extensions of the two specific examples shown above. For example in embodiment $\mathrm{B}$, other heat conducting metal frames could be used, the gap or moat meant to isolate the slab thermally and mechanically from the frame could by wider than $2 \mathrm{~mm}$, other mechanisms of blackening could be used, etc.

The success of these two embodiments in suppressing ASE and preventing laser gain from clamping at levels that would negatively effect our laser performance, as well as the actual SSHCL performance with slabs so constructed is documented in several EXCEL spreadsheets and other data files by Scott Fochs and Mark Rotter. Other specifics on the various bonding agents, etc. that we have tried can also be made available either by myself or Steve Letts. 